\title{
ELECTRON DYNAMICS IN THE HERA LUMINOSITY UPGRADE LATTICE OF THE YEAR 2000
}

\author{
G. Hoffstätter ${ }^{*}$, F. Willeke, DESY, Hamburg, Germany
}

\section{Abstract}

It is planned to upgrade the HERA luminosity to $7 \cdot 10^{31} \mathrm{~cm}^{-2} \mathrm{~s}^{-1}$, which is 4 times the original design luminosity. This is to be achieved by decreasing the proton beam size by moving quadrupoles closer to the interaction point and by increasing their strength. Similar measures decrease the electron beam size. However, to match the smaller proton beam size, the horizontal electron emittance additionally has to be decreased.

The electron emittance can be decreased either by stronger focusing in the arcs, or by changing the damping partition numbers. In the HERA case, however, both methods have to be applied simultaneously, since changing the damping partition numbers increases the longitudinal emittance, which can only be tolerated with the current RF parameters if the bucket is increased by a stronger focusing in the arcs. These two methods of decreasing the emittance have competing effects on long term stability. Stronger focusing usually leads to a greater reduction of the dynamic aperture than of the emittance; whereas a change of the damping partition numbers tends to increase the dynamic aperture relative to the emittance. Playing the two competing effects against each other, it is possible to decrease the electron emittance while keeping the relative dynamic aperture as well as the requirements on the RF system tolerable.

\section{EMITTANCE REDUCTION}

In the HERA luminosity upgrade the horizontal emittance of the electron beam has to be reduced from currently $41 \pi \mathrm{nm}$ to $22 \pi \mathrm{nm}[1,2]$. The horizontal emittance $\varepsilon_{x}$ of an electron storage ring is given by

$$
\begin{aligned}
\varepsilon_{x} & =\frac{C_{q} \gamma^{2}}{1-\mathcal{D}} \frac{<|G|^{3} \frac{1}{\beta}\left[\eta^{2}+\left(\beta \eta^{\prime}+\alpha \eta\right)^{2}\right]>}{<G^{2}>}, \\
\mathcal{D} & =\frac{\left\langle\eta G\left(G^{2}+2 K\right)>\right.}{<G^{2}>},
\end{aligned}
$$

with the curvature $G$ of and the focusing strength $K$ on the closed orbit. The parentheses $\langle\ldots\rangle$ indicate an average around the ring and $\eta$ is the periodic dispersion. The optic functions $\alpha$ and $\beta$ are used and $C_{q} \approx 384 \mathrm{fm}$ is a constant. On the design orbit of a separated function ring, which HERA is to a good approximation, $G \cdot K=0$ around the ring.

Since the curvature in dipoles is fixed, there are two ways of reducing the emittance: changing $\mathcal{D}$ in the denominator by shifting the RF frequency and changing the numerator by focusing stronger in the FODO cells. An RF frequency

\footnotetext{
*Email: georg.hoffstaetter@desy.de
}

shift changes the energy of the electrons and makes the beam travel along a dispersive orbit, which has curvature in quadrupoles. The product $G \cdot K$ no longer vanishes in quadrupoles, and changes in $\mathcal{D}$ of the order of one can easily be obtained which largely changes the horizontal emittance. changed. However, the energy spread $\sigma_{\delta}$ of the electron bunch increases when $\varepsilon_{x}$ is decreased by this method since it is determined by

$$
\sigma_{\delta}^{2}=\frac{C_{q} \gamma^{2}}{2+\mathcal{D}} \frac{<|G|^{3}>}{\left\langle G^{2}>\right.} .
$$

If one does not want to compromise on particle loss out of the RF bucket, the RF bucket height $\Delta E / E_{0}$ has to be increased accordingly. We do not want to increase the bucket size by increasing the cavity voltage, since we need all available power for the storage of the $56 \mathrm{~mA}$ design current. Therefore we have to decrease the dispersion to take advantage of the fact that $\Delta E / E_{0} \propto 1 / \sqrt{\langle G \eta\rangle}$. The dispersion $\eta$ in the arcs of the ring is decreased when we increase the horizontal focusing from currently $60^{\circ}$ per FODO cell. By doing so, an additional fact comes in very handy: stronger focusing reduces the emittance by reducing the numerator of equation 1.

\section{DYNAMIC APERTURE}

Initially it was tried to obtain the $22 \mathrm{~nm}$ design emittance by stronger focusing alone [3]. This could be achieved by going to $90^{\circ}$ horizontal phase advance per FODO cell. However, the stronger natural chromaticity required stronger sextupoles and these nonlinear fields reduced the dynamic aperture significantly. The dynamic aperture for on energy particles for the four slightly different electron optics used in HERA during 1997 and 1998 are shown in figure 1. These calculations were performed by tracking for 1000 turns with MAD. Tracking with the second order TRANSPORT formalism and with third and fourth order generating functions leads to equivalent results. The dynamic aperture was always in a band between the $23 \sigma$ and the $31 \sigma$ horizontal and vertical emittance. The vertical emittance was assumed to be $\varepsilon_{x} / 2$, which has proved to be a useful assumption in the literature. The dynamic aperture for the lumi upgrade optics with $90^{\circ}$ horizontal phase advance per FODO cell is reduced to approximately $15 \sigma$ and is also shown in this figure.

Because of this disadvantage of unnecessary strong focusing we have analyzed the possibility of reducing the emittance partly by stronger focusing and partly by an RF frequency shift. Figure 2 shows the decrease of the equilibrium emittance with an RF frequency increase and with an increase of horizontal focusing. On the red line, the ratio 


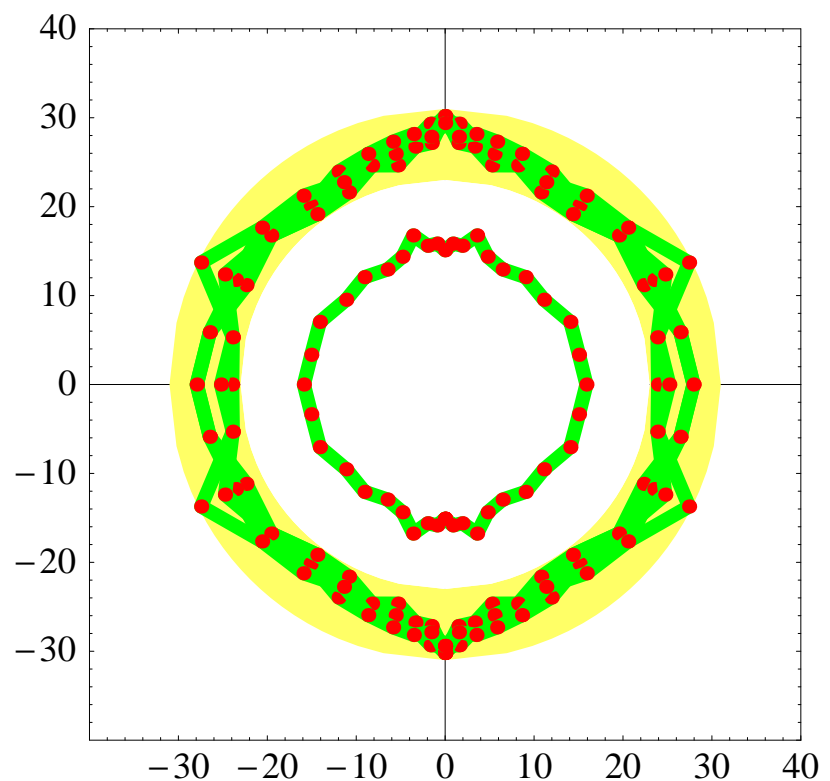

Figure 1: The boundaries or stable motion $x$ and $y$ are shown in units of beam sigmas. The dynamic apertures between $23 \sigma$ and the $31 \sigma$ were computed for the four electron optics used in HERA during 1997 and 1998. The curve around $15 \sigma$ was computed for a lumi upgrade optics with $90^{\circ}$ phase advance per FODO cell, which leads to the $22 \mathrm{~nm}$ design emittance.

of relative bucket height to energy spread is constant. For a given phase advance we should therefore not increase the RF frequency further than indicated by the red line. However, we also do not want to shift it less, since this would imply unnecessary strong focusing. For the lumi upgrade lattice, the point on the red curve which leads to the $22 \mathrm{~nm}$ design emittance is very close to $72^{\circ}$ phase advance and an RF frequency increase of $200 \mathrm{~Hz}$ in HERA's $500 \mathrm{MHz}$ system.

Without an RF frequency shift, the electron beam in the new $72^{\circ}$ lattice has an emittance of $27 \pi \mathrm{mm} \cdot \mathrm{mrad}$ at $27.5 \mathrm{GeV}$. The stronger focusing gives space for $200 \mathrm{~Hz}$ increase in frequency which further reduces the emittance to $22 \pi \mathrm{mm} \cdot \mathrm{mrad}$. The energy decrease going along with such a frequency shift is only $-0.083 \%$. The energy dependent dynamic aperture of this new lumi upgrade lattice is shown in figure 3 .

\section{CHROMATIC OPTICS CORRECTION}

The on energy dynamic aperture of the presented lumi upgrade optics is as large as the current dynamic aperture. The reduction of the dynamic aperture with energy deviation can possibly be reduced by chromatic sextupole correction of the optics.

At present six independent families of sextupoles are used in the $60^{\circ}$ lattice to reduce the sensitivity of the beta functions and dispersion on energy. There are two sextupoles in each FODO cell and every sixth sextupole in

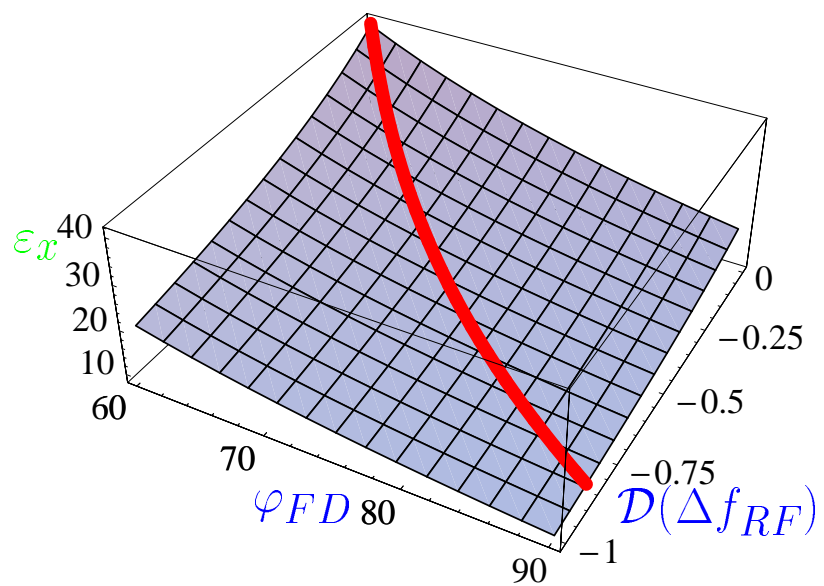

Figure 2: The change of the horizontal emittance with focusing per FODO cell and with $\mathcal{D}$. On the read line the ratio of energy bucket size to energy spread of the beam is invariant; here the focusing is just strong enough to allow for the RF frequency shift. This red curve leads to a $22 \mathrm{~nm}$ emittance at $72^{\circ}$ focusing.

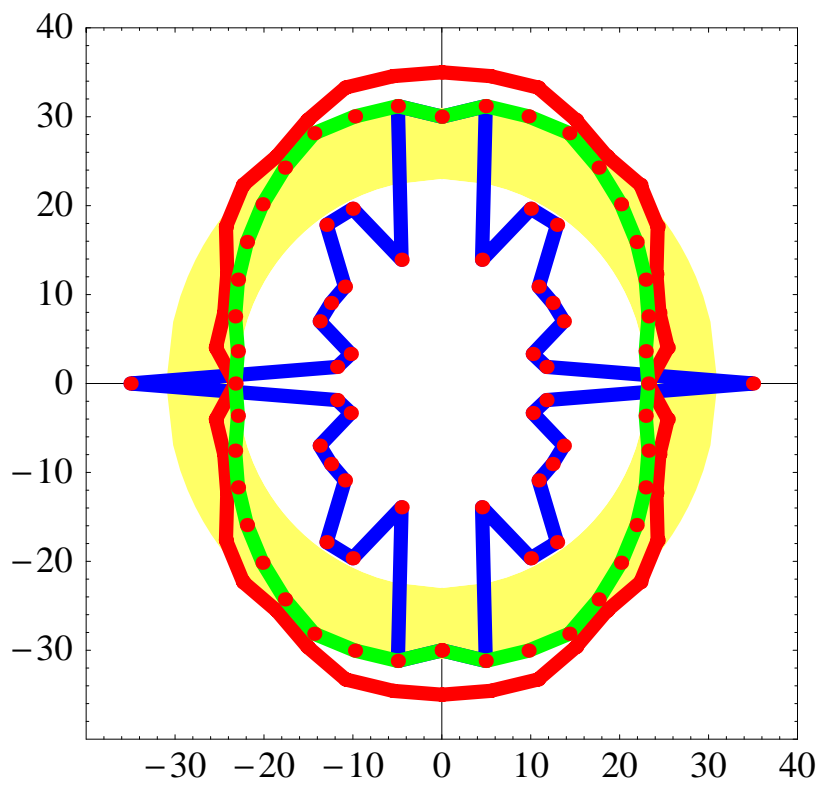

Figure 3: The boundaries of stable motion in $x$ and $y$ shown in units of beam sigmas for the $72^{\circ}$ lumi upgrade optics with RF frequency shift for energy deviations (red: $-0.25 \%$, green: $-0 \%$, blue: $0.25 \%$ ).

one octant belongs to the same family. In this interleaved sextupole arrangement the betatron phase advance between successive elements of one families is $180^{\circ}$, and therefore the sextupoles of a family have a coherent effect on the chromatic beta beat. Each octant has 24 FODO structures with sextupoles and therefore 8 sextupoles for each family.

In a $72^{\circ}$ optics the phase increases by $180^{\circ}$ after every 5th FODO and we are therefore left with ten independent families. If two sextupoles are added to each octant, each family has 5 sextupoles per octant. Since there are 
currently only wires and power supplies for six independent families, we have fitted only six independent sextupole field strength to minimize the chromatic beta and dispersion beats. One can freely choose which of the 5 families to keep dependent and which to optimize independently.

\section{FEASIBILITY OF AN RF FREQUENCY SHIFT IN HERA}

There is a leverage for decreasing the emittance by increasing the RF frequency only if the current operation RF frequency $f_{0}$ is not too far above the central frequency $f_{c}$, where $\mathcal{D}$ in equation 1 is approximately zero. Several experiments were therefore performed during the December 1998 machine studies to specify a possible shift of the current $\mathrm{RF}$ frequency away from $f_{c}$ [4].

The current frequency $f_{0}$ of the RF system is known very accurately. The central frequency $f_{c}$, however, is defined by the length of the specific closed orbit which goes through the center of quadrupoles; and five methods were applied to measure this frequency. The results together with an estimate of the random errors of these methods are shown in table 1 . They all show that the current frequency $f_{0}$ is not severely above $f_{c}$; more likely $f_{0}$ is even below $f_{c}$. The frequency can therefore safely be increased to reduce the horizontal emittance in the luminosity upgrade project.

Table 1: The distance of HERA's operation RF frequency from the central frequency

\begin{tabular}{|l|c|}
\hline Measurement & $\Delta f_{0}=f_{0}-f_{c}$ \\
\hline Beam loss at damping poles & $-163 \pm 20 \mathrm{~Hz}$ \\
Extrapolation of damping rates & $-250 \pm 150 \mathrm{~Hz}$ \\
Horizontal center of sextupoles & $+130 \pm 25 \mathrm{~Hz}$ \\
Vertical center of sextupoles & $-70 \pm 50 \mathrm{~Hz}$ \\
Emittance change with frequency & $-175 \pm 70 \mathrm{~Hz}$ \\
\hline
\end{tabular}

\section{FEASIBILITY OF STRONGER FOCUSING IN HERA}

To test the usability of a $72^{\circ}$ electron optics in the upgraded HERA, a $72^{\circ}$ optic was installed in the current ring, which leads to a simulated horizontal emittance of $34 \pi \mathrm{nm}$. The dynamic aperture of this optics was measured and compared to measurements of the dynamic aperture for the current $60^{\circ}$ optics.

The sextupoles in this new $72^{\circ}$ luminosity optics and in the $72^{\circ}$ injection optics were used in two families to correct the chromaticities. Since the current six family scheme is designed for a $60^{\circ}$ optics, no chromatic correction of optical functions was performed in the $72^{\circ}$ case.

With these new optics arrangements injection, accumulation, ramp, and installing the luminosity optics and tunes of $\mathrm{Qx}=52.148$ and $\mathrm{Qy}=52.216$ was unproblematic. The usual lifetime of around 15 hours at low currents (15mA) could be achieved and the orbit could be corrected to a usual rms of $1.22 \mathrm{~mm}$ horizontally and 0.98 vertically. For this condition the dynamic aperture was then measured and compared with old measurements for the current $60^{\circ}$ luminosity optics.

\subsection{Measurement of dynamic aperture}

When the beam is kicked by an angle $\Theta$, the central particles travel with a Courant Snyder invariant $\beta_{k} \Theta^{2}$, with the beta function $\beta_{k}$ at the kicker. When the central particles are kicked to a Courant Snyder invariant which corresponds to the dynamic aperture $D A$, then approximately half of the beam distribution will be outside the dynamic aperture and half will be inside. The kick which leads to a loss of half the beam was measured at $27.5 \mathrm{GeV}$; the absolute dynamic aperture (DA) and the DA relative to the computed emittances is depicted in table 2 .

Table 2: Dynamic aperture for the $72^{\circ}$ and the $60^{\circ}$ optics in the current HERA. The dynamic apertures are comparable, but both are presumably small due to defect magnets, which have by now been repaired.

\begin{tabular}{|l|c|c|}
\hline Optics & $D A$ & $D A_{\text {rel }}$ \\
\hline $72^{\circ}$ luminosity & $2.7 \pi \mathrm{mm} \cdot \mathrm{mrad}$ & $8.5 \sigma$ \\
$72^{\circ}$ ramp file & $2.6 \pi \mathrm{mm} \cdot \mathrm{mrad}$ & $8.1 \sigma$ \\
$60^{\circ}$ luminosity \# 1 & $2.6 \pi \mathrm{mm} \cdot \mathrm{mrad}$ & $7.9 \sigma$ \\
$60^{\circ}$ luminosity \# 2 & $2.2 \pi \mathrm{mm} \cdot \mathrm{mrad}$ & $7.2 \sigma$ \\
$60^{\circ}$ luminosity \# 3 & $2.3 \pi \mathrm{mm} \cdot \mathrm{mrad}$ & $7.4 \sigma$ \\
$60^{\circ}$ luminosity \# 4 & $2.4 \pi \mathrm{mm} \cdot \mathrm{mrad}$ & $7.6 \sigma$ \\
\hline
\end{tabular}

On average the dynamic aperture was $7.5 \sigma$ with the current $60^{\circ}$ optics; with the $72^{\circ}$ optics the average was $8.3 \sigma$. Since these studies, shortages to ground were found in three magnets. The repair of these shortages increased the injection efficiency strongly and most likely the dynamic aperture has also increased, future measurements will show by how much.

The fact that the dynamic aperture did not decrease when installing the new optics supports the applicability of a $72^{\circ}$ optics in the luminosity upgrade.

\section{REFERENCES}

[1] Editor: U. Schneekloth, "The HERA Luminosity Upgrade”, DESY-HERA 98-05 (1998)

[2] M. Seidel for the HERA upgrade group, "Luminosity Upgrade of HERA", these proceedings (1999)

[3] T. Sen, "Dynamic aperture in the luminosity upgrade HERAe lattice", EPAC Proceedings, Stockholm and DESY M 98-06 (1998)

[4] M. Bieler et al., "HERA machine studies December 1998", DESY M 99 (1999) 